\title{
Dependence of Cadmium Sulfide Fluorescence on the Excitation Light Energy
}

\author{
Vyacheslav N Pak*, Sergey M Shilov and Yulia Yu Gavronskaya \\ Department of Chemistry, Herzen State Pedagogical University of Russia, Russia
}

Received: 䟱January 25, 2018; Published: 制 February 15, 2018

*Corresponding author: Vyacheslav N Pak, Department of Chemistry, Herzen State Pedagogical University of Russia, Russia; Email: pakviacheslav@mail.ru

\begin{abstract}
Monotonic dependence of the fluorescence band position on the exciting light energy has been shown in polycrystalline hexagonal cadmium sulfide at $293 \mathrm{~K}$. The observed phenomenon is interpreted on the basis of a qualitative model assuming the emission levels initiation in the energy gap as a result of antibonding (Cd-S)*-orbital's occupation in the course of electron excitation.
\end{abstract}

Keywords: Cadmium sulfide; Luminescence

\section{Introduction}

Cadmium sulfide will be shown below as a good example giving opportunity to observe monotonic shift of its fluorescence spectrum as a result of the excitation light energy variation. It is worthy to note that an assumption concerning a probability of the steady dependence of the fluorescence band maximum position $\mathrm{I}_{\mathrm{f}}$ on the exciting light wave length $\mathrm{I}_{\mathrm{e}}$ can be formulated on the qualitative basis for the substances of specific electron structure. In fact, complicated fluorescence spectra are sometimes observed with their components (bands) being activated by a different exciting light wave length [1-5] thus comprising a "discrete" variant of $\mathrm{I}_{\mathrm{f}}$ dependence on $\mathrm{I}_{\mathrm{e}}$ which specific character is caused by a presence of several long-living excited states. If one admits further an existence of a solid in which these states are so numerous that form a band, such an object has a good chance to exhibit a continuous variant of the $I_{f}$ dependence on $I_{e}$. In other words, a possibility of the proposed fluorescence centers distribution on the energy scale may be regarded as limiting case that can possibly take place in some solids, CdS being one of them.

\section{Experimental}

The experimental results were obtained from the measurements of polycrystalline CdS sample of hexagonal (vourtzite) modification qualified as "particularly pure" (VNIIL, Stavropol, Russia). Comparative spectra measurements were specially performed with several other CdS samples of high purity with either different industrial prehistory or prepared in laboratory. All of them showed close similarity of their optical properties. Fluorescence spectra registration was carried out at $293 \mathrm{~K}$ with the use of automatic spectrometer SDL-1 (this and other optical instruments was developed by LOMO-Association, SaintPetersburg, Russia). The excitation was performed in the region of CdS fundamental absorption $\mathrm{I}_{\mathrm{e}}=500 \div 300 \mathrm{~nm}$. A wave length of the exciting irradiation was set by intensive light monochromator MDR-12 while xenon lamp LXW (150 Wt) served as a light source. Impulse $\tau$-meter TM-12 was used to determine a life time of the excited states in CdS which turned out to be in the range $\tau^{*}=$ $40 \div 50 \mu$ s for all the measurements. In order to detail the $I_{f}$ and $I_{e}$ correlation the excitation spectra were also obtained by measuring the fluorescence intensities at fixed wavelengths in visible region while the excitation wavelength was varied continuously.

\section{Results and Discussion}

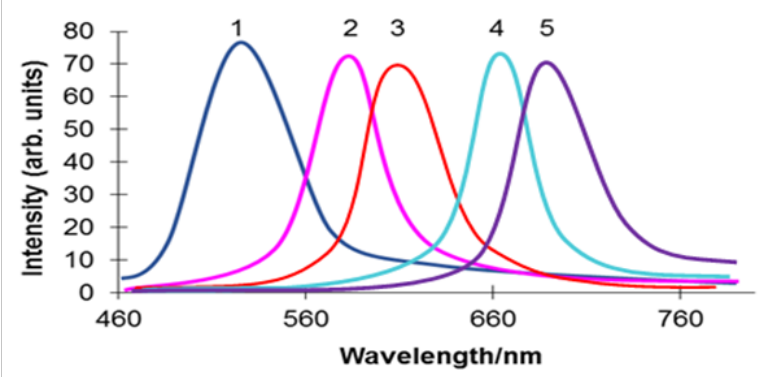

Figure 1: CdS fluorescence spectra corresponding to the excitation monochromatic light of wave length $\mathrm{I}_{\mathrm{e}}(\mathrm{nm}): 300$ (1), $337(2), 390(3), 440(4)$ and $510(5)$. 
Figure 1 shows a selected row of the fluorescence CdS spectra confirming the dependence of $\mathrm{I}_{\mathrm{f}}$ on $\mathrm{I}_{\mathrm{e}}$. Monochromatic exciting light of the wavelength within the region of $\mathrm{CdS}$ fundamental absorption produces the only individual fluorescence band with a maximum corresponding to the excitation energy. The inspection of wide optical interval, as well as special measurements performed with a short $I_{e}$ steps, confirmed the distinctly registered shifts in CdS fluorescence spectrum, thus, providing the dependence of simbat and close to continuous character. The increase of $I_{e}$ from 300 to $510 \mathrm{~nm}$ causes the significant shift of the $\mathrm{I}_{\mathrm{f}}$ maximum from green to red region $(530 \rightarrow 700 \mathrm{~nm})$. Gaussian shapes, as well as similar intensities of the fluorescence bands, are observed in all cases giving some opportunities for the assumption about the same origin of the numerous excited states and the uniformity of the energy band they form. The excitation fluorescence spectra also demonstrate a correspondence of wavelengths $\lambda_{\mathrm{e}}$ and $\lambda_{\mathrm{f}}$ (Figure 2). For example, the excitation bands with maxima $\lambda_{\mathrm{e}}$ at $390 ; 440$; $510 \mathrm{~nm}$ are responsible for the emission at $\lambda_{\mathrm{f}}=605 ; 660 ; 700 \mathrm{~nm}$ etc. A graphical form of the established dependence is represented by Figure 3 in the coordinates of the wave numbers.

The observed spectral peculiarities apparently cannot be attached to the commonly accepted admixture and/or defect model of the photo-fluorescent solids [1-5]. In this connection it is worthy to draw attention to the specific feature of cadmium ionic (and atomic) electron configuration, namely, deeply lying occupied $4 \mathrm{~d}^{10}$-levels. This feature results in the extremely important consequence for the CdS behavior under excitation that is the electrons transitions to the antibonding (Cd-S)* states (Figure 4). This statement itself does not seem to need any proof since there are no other orbital's which can receive the electrons in CdS. It is pertinent to note here that quite another situation is represented by a great majority of other d-metals of 4,5,6 periods (except Zn and $\mathrm{Hg}$ ) in their compounds due to the role of the d-orbital's as the electron acceptors in excited state. These orbital's are partially occupied (or vacant) and separated with the forbidden bandgap from the electron-donor nonbonding occupied p-orbital of nonmetals (sulfur, oxygen etc.). As a result, interband electrons excitations in these substances are known as charge-transfer from $\mathrm{p}_{\text {(nonmetal) }}$ to $\mathrm{d}_{\text {(transition metal) }}$ levels [6]. It should be emphasized that high energy antibonding states are unattainable in this cases.

It is nearly evident that sulfur nonbonding 3p-orbitals are the highest occupied in CdS, thus, forming the upper border of the valence band. In order to evaluate a relative position of the narrow energetic strip formed by sulfur 3p-levels, toward cadmium $4 \mathrm{~d} 10$, the atomic valence state ionization potentials may be used as the first approximation [6]. The corresponding value for sulfur ground state $\mathrm{E}_{3 \mathrm{p}}\left(3 \mathrm{~s}^{2} 3 \mathrm{p}^{4}\right)=-10.36 \mathrm{eV}$ in comparison with cadmium ionization potential $\mathrm{E}_{4 \mathrm{~d}}\left(4 \mathrm{~d}^{10} 5 \mathrm{~s}^{1} 5 \mathrm{p}^{1}\right)=-17.16 \mathrm{eV}$ give good opportunities to look at sulfur nonbonding $3 p$-orbitals as highest occupied in CdS. Therefore, the electron transitions $3 \mathrm{p}(\mathrm{S}) \rightarrow(\mathrm{Cd}-$ $S)^{*}$ take place when CdS is irradiated with the light of the energy above its energy gap. It is the antibonding orbitals occupation which moves to the assumption that the reason of the electrons "delay" in the excited states (i.e. its life time increase) lies in the weakening of $\mathrm{Cd}-\mathrm{S}$ bonds that can be looked at as a real local perturbation of CdS structure under irradiation. It is our hypothesis that the lowering of Cd-S bonds strength in excited state causes a shift of corresponding (Cd-S)*-levels into forbidden band followed by subsequent electron transitions $(\mathrm{Cd}-\mathrm{S})^{*}-3 \mathrm{p}(\mathrm{S})$ with the energy less than needed for the fluorescence excitation (Figure 4).

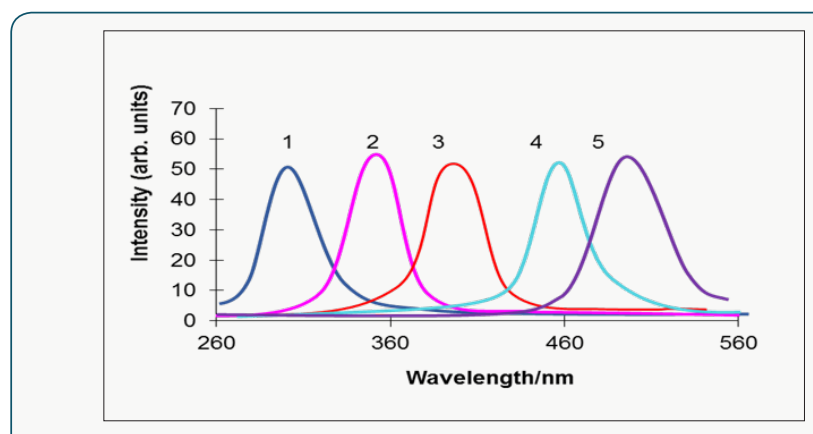

Figure 2: CdS excitation spectra corresponding to the fluorescence bands maxima $\lambda_{\mathrm{f}}(\mathrm{nm}): 530$ (1), 560 (2), 605 (3), 660 (4) and 700 (5).

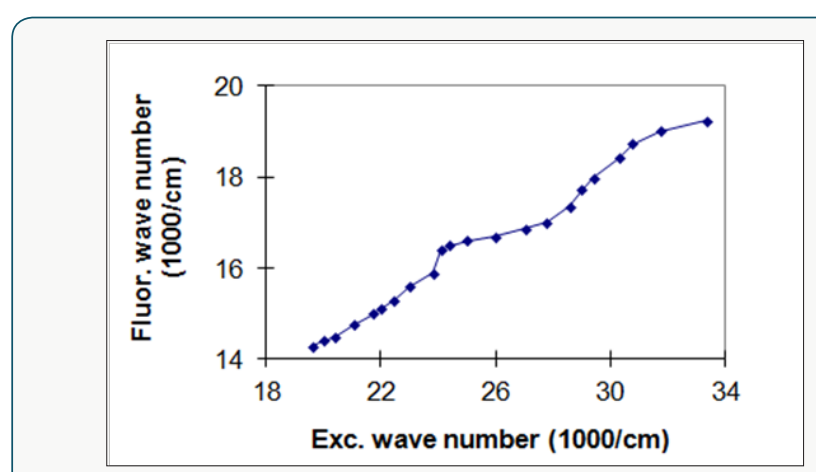

Figure 3: The relation between fluorescence and excitation bands maxima in $\mathrm{CdS}$.

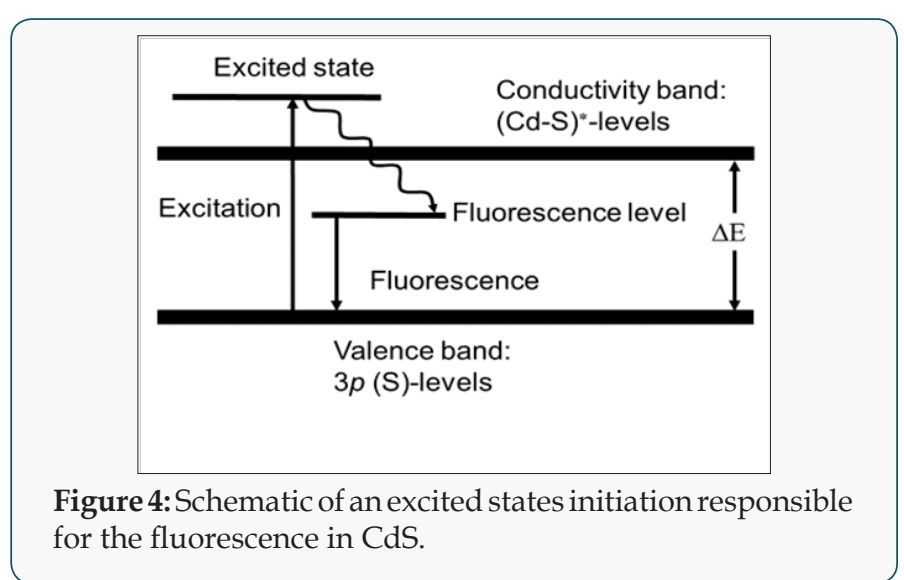

Irradiation and emission acts in CdS are, therefore, reasonably separated in time which is needed for the structural relaxation of light-induced distortions. Based on this model, a continues increase of the exciting light energy leads to the occupation of increasingly 
higher (Cd-S)*- levels that consequently results in the emission states shift away from sulfur 3p-orbitals (Figure 4) in qualitative agreement with the experimental data (Figures 1-3). It is worthy to note that $\mathrm{CdS}$ fluorescence behavior revealed and discussed here does not appear in the case of $\mathrm{ZnS}$, being electronically analogous. In fact $\mathrm{ZnS}$ comprises well known system demonstrating the fluorescence severe dependence on purity, stoichiometry and preparative conditions [4,5]. In order to understand CdS and $\mathrm{ZnS}$ optical dissimilarities a short analysis of their structure-chemical differences based on data seems to be helpful. More than 150 poly types which comprise nonequilibrium intermediates formed by the mixing of the sphalerite and vourtzite structures are known for $\mathrm{ZnS}$-system. The rhomboedric modification of $\mathrm{ZnS}$ as well as polysulfide's ZnSx, are also well-known.

So-called martensite-type transformations take place in $\mathrm{ZnS}$ when the increasing of the defects concentration causes the distortion of lattice parameters. Being typical for $\mathrm{ZnS}$, these very special features are quite unknown in the case of CdS. The principle difference of CdS and $\mathrm{ZnS}$ may be further illustrated by the fact that $\mathrm{ZnS}$ evaporation is accompanied by its dissociation whereas CdS sublimates and forms stable poly molecular ensembles in gasphase. In addition to all above mentioned, CdS is well-known, in general, as much more stable toward a dissolution, acids action, oxidation and reduction than $\mathrm{ZnS}$. Being taken together these facts qualify ZnS system as very changeable and reactive, leading further to the assumption that the great majority of $\mathrm{ZnS}$ samples are really nonhomogeneous from structural point of view. This assumption, in turn, implies the nonhomogeneity of $\mathrm{ZnS}$ conduction band composed of (Zn-S)*-states (corresponding to $\mathrm{Zn}-\mathrm{S}$ bonds of a different strength). Therefore, in the case of ZnS irradiation, the excitation energy is partially spent to weaken one or several types of the most unstable bonds that results in the formation of one or several emission states well determined for each sample and independent on excitation energy.

\section{Conclusion}

Finally, there is a good reason to believe that the monotonic dependence of the fluorescence band position on excitation energy in the case of CdS is determined by a compliance with two conditions, namely, the occupation of antibonding (Cd-S)*-states under excitation as well as homogeneity of the conduction band they form. Thus, it is not excluded in principle that CdS fluorescence peculiarity will turn to be intrinsic for some other solid compounds. Their number, however, may be restricted by a necessity of the two above mentioned conditions to be met.

\section{Acknowledgment}

This work was supported by the Ministry of Education and Science of Russia within the base part of the Government Instruction.

\section{References}

1. Ronda C (2007) Luminescence: From Theory to Applications. Wiley, New York, USA.

2. Sharma A, Schulman SJ (1999) Introduction to fluorescent spectroscopy. Wiley, New York, USA.

3. Lakowicz JR (1983) Principles of fluorescence spectroscopy. Plenum, New York, USA.

4. Samsonov GV, Drozdova SV (1972) Sulfides. Metallurgia, Moscow, Russia.

5. Morozova NK, Kuznetsov VA (1987) Zinc sulfide: Preparation and optical properties. Nauka, Moscow, Russia.

6. Bersuker IB (1996) Electronic Structure and Properties of Transition Metals Compounds. Wiley, New York, USA.
CC (i) This work is licensed under Creative Commons Attribution 4.0 License

To Submit Your Article Click Here: Submit Article DOI: $10.32474 /$ AOICS.2018.01.000116

\section{AOICS}

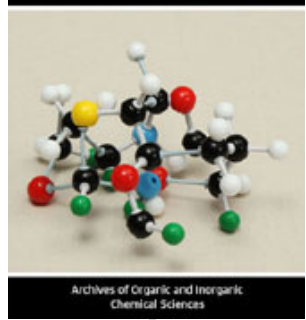

Archives of Organic and Inorganic Chemical Sciences

\section{Assets of Publishing with us}

- Global archiving of articles

- Immediate, unrestricted online access

- Rigorous Peer Review Process

- Authors Retain Copyrights

- Unique DOI for all articles 